\title{
On the relation of three theorems of analysis to the axiom of choice
}

\author{
ADRIAN F D FELLHAUER
}

\begin{abstract}
In what follows, essentially two things will be accomplished. First, it will be proven that a version of the Arzelà-Ascoli theorem and the Fréchet-Kolmogorov theorem are equivalent to the axiom of countable choice for subsets of real numbers. Secondly, some progress is made towards determining the amount of axioms that have to be added to the Zermelo-Fraenkel system so that the uniform boundedness principle holds.
\end{abstract}

2010 Mathematics Subject Classification 03B30, 03E25 (primary); 46B25, 46B50, 46E15, 46E30 (secondary)

Keywords: real analysis, functional analysis, axiom of choice, Arzelá-Ascoli, Fréchet-Kolmogorov, Uniform boundedness principle

\section{Introduction}

\subsection{Outline}

In Subsection 1.2, we briefly describe the greater goal of this paper.

In Section 2, we treat the theorems of Arzelà-Ascoli and Fréchet-Kolmogorov in the following manner: In Subsection 2.1, we state precisely the theorems which we will investigate and briefly comment on them, in Subsection 2.2 we prove modified versions of both theorems that hold without any choice axiom, and using these, in Subsection 2.3 we prove that both theorems under consideration are equivalent to the axiom of countable choice for subsets of real numbers.

In Section 3, we investigate the uniform boundedness principle as follows: In Subsection 3.1 we state the uniform boundedness principle, clarify how it relates (in ZF) to several other theorems and state the knowledge regarding its choice-axiomatic strength until this paper, in Subsection 3.2 we give a new proof of the fact that the uniform boundedness principle follows from the axiom of countable choice, in Subsection 3.3 we deduce several choice-like axioms from the uniform boundedness principle, in Subsection 3.4 
we give a weak version of the uniform boundedness principle which is equivalent to the axiom of countable multiple choice, and in Subsection 3.5 we elaborate on how our results are incomplete and what seem promising directions for further investigation.

\subsection{Context and motivation}

Many mathematicians blindly accept the axiom of choice. This may be because it is a straightforward generalisation of things which hold trivially in the finite to the infinite. However, history has proven (for instance in the case of summation) that such generalisations may yield contradictions. Now for the axiom of choice, the situation is somewhat different because it is logically independent of the Zermelo-Fraenkel system (or for short: ZF); from this follows that ZF plus the axiom of choice can only lead to a contradiction if $\mathrm{ZF}$ already leads to a contradiction. However, if physical reality is used as a model for the axioms that are used, the Banach-Tarski paradox doubtlessly contradicts the preservation of energy and mass (since the amount of energy used in reassembling an object of a certain sufficient size is surely far lower than the energy needed for creating an entire object of the same size).

If the axiom of choice is not accepted, an alternative approach may be, instead of proving theorems for all, say, Banach spaces or rings or whatever object, to only prove these theorems for classes of spaces which are defined such that certain choice-axiomatic properties hold for them. For instance, the proof of Theorem 3.2.1 and the ensuing remark will demonstrate that one may prove the uniform boundedness principle in $\mathrm{ZF}$ for all Banach spaces in which every countable product of open subsets is nonempty. But it is most certainly sufficient to assume that all countable products of any sets are nonempty (the axiom of countable choice).

In this context, the aim of this paper is to advance knowledge on exactly how much choice is needed to hold for a given space so that certain theorems are true.

\section{The Arzelà-Ascoli and Fréchet-Kolmogorov theorems}

\subsection{Introduction}

When $X$ is a topological space and $Y$ is a metric space with metric $d_{Y}$, the set of continuous, bounded functions from $X$ to $Y$ with metric

$$
d(f, g):=\sup _{x \in X} d_{Y}(f(x), g(x)) .
$$


is a metric space which is denoted by $\mathcal{C}(X, Y)$. We write $\mathcal{C}(X)$ for $\mathcal{C}(X, \mathbb{R})$.

There are several closely related results that bear the name 'Arzelà-Ascoli theorem' (see for instance Brezis [6, Theorem 4.25, page 111], Rudin [20, Theorem 7.25, page 158], Tao [23, Theorem 1.8.23, page 114], or Yosida [25, page 85ff.]); all concern (relative) compactness in spaces of continuous functions. We shall be concerned with the following version:

2.1.1 Theorem (Arzelà-Ascoli) Let $K \subset \mathbb{R}^{d}$ be compact (bearing the topology on $K$ that is induced by the Euclidean topology of $\mathbb{R}^{d}$ ) and let $\mathcal{F} \subseteq \mathcal{C}(K)$. Then the following two are equivalent:

(1) Every sequence in $\mathcal{F}$ contains a convergent subsequence.

(2) $\mathcal{F}$ is uniformly bounded and equicontinuous.

We will prove that Theorem 2.1.1 is equivalent to the axiom of countable choice for subsets of the real numbers. (Note that other versions of the Arzelà-Ascoli theorem have also been studied with regard to their axiomatic strength, eg in Herrlich [11].)

The Fréchet-Kolmogorov theorem concerns (relative) compactness in certain $L^{p}$ spaces; it is contained within several (perhaps most) introductory functional analysis textbooks (for instance in Brezis [6, Theorem 4.26, page 111] or Yosida [25, page 275]).

2.1.2 Theorem (Fréchet-Kolmogorov) Let $1 \leq p<\infty$, let $S \subset \mathbb{R}^{d}$ be bounded and measurable and let $\mathcal{F} \subseteq L^{p}(S)$. Then the following are equivalent:

(1) Every sequence in $\mathcal{F}$ contains a convergent subsequence.

(2) $\mathcal{F}$ is bounded in $L^{p}(S)$ and

$$
\lim _{h \rightarrow 0} \sup _{f \in \mathcal{F}} \int_{S}|f(x+h)-f(x)|^{p} d x=0 .
$$

Note that for the integral in the above limit to make sense, $f \in \mathcal{F}$ is extended to $\mathbb{R}^{d}$ by being zero outside $S$.

\subsection{Modified versions of both theorems}

As a first step of investigating the choice-axiomatic nature of the Arzelà-Ascoli and Fréchet-Kolmogorov theorems, we establish modified versions of both theorems which hold true in ZF, without assuming any version of the axiom of choice. Oddly enough, these modified versions will later be needed in determining the choice-axiomatic strength of the full theorems of Arzelà-Ascoli and Fréchet-Kolmogorov as given above. 
2.2.1 Theorem (Modified Arzelà-Ascoli theorem) Let $X$ be a compact ${ }^{1}$ and separable ${ }^{2}$ metric space with metric $d_{X}$, let $Y$ be a metric space with metric $d_{Y}$ and let $\mathcal{F} \subseteq \mathcal{C}(X)$. Then the following two are equivalent:

(1) Every sequence in $\mathcal{F}$ contains a convergent subsequence.

(2) Every countable subset of $\mathcal{F}$ is pointwise relatively sequentially compact and equicontinuous.

Proof For $(2) \Rightarrow(1)$, the proof given in Yosida [25, page 85ff.] is essentially sufficient; note only that

- the Bolzano-Weierstraß theorem may be proven constructively (see for instance Abbott [1, Theorem 2.5.5, page 64]), and

- when $\left\{s_{n} \mid n \in \mathbb{N}\right\}$ is a countable, dense subset of $X$, then for every $\epsilon>0$ there must automatically exist (a minimal) $k(\epsilon)$ such that

$$
\sup _{s \in S} \inf _{1 \leq j \leq k(\epsilon)} d\left(s_{j}, s\right) \leq \epsilon
$$

this follows by considering the open cover $\left\{B_{\epsilon}\left(s_{n}\right) \mid n \in \mathbb{N}\right\}$ and applying compactness.

For $(1) \Rightarrow(2)$, one may consult the proof given in Tao [23, Proof of Theorem 1.8.23, (i) $\Rightarrow$ (ii), page 114]; note only that in a separable space, a countable dense subset $\left\{x_{n} \mid n \in \mathbb{N}\right\}$ yields a choice function on the set of all open sets, for one may take the first $x_{n}$ contained within a given open set.

2.2.2 Theorem (Modified Fréchet-Kolmogorov theorem) Let $1 \leq p<\infty$, let $S \subset \mathbb{R}^{d}$ be bounded and measurable and let $\mathcal{F} \subseteq L^{p}(S)$. Then the following are equivalent:

(1) Every sequence in $\mathcal{F}$ contains a convergent subsequence.

(2) Every countable $\mathcal{G} \subseteq \mathcal{F}$ is bounded in $L^{p}(S)$ and satisfies

$$
\lim _{h \rightarrow 0} \sup _{f \in \mathcal{G}} \int_{S}|f(x+h)-f(x)|^{p} d x=0 .
$$

\footnotetext{
${ }^{1}$ By 'compact' we mean 'every open cover has a finite subcover.' Note that with this definition the statement 'A pseudometric space is compact if and only if it is sequentially compact' is equivalent to the axiom of countable choice (see Bentley and Herrlich [2, Theorem 4.3, page 161]).

${ }^{2}$ Note that the assertion 'every compact pseudometric space is separable' is equivalent to the axiom of countable choice (see Bentley and Herrlich [2, Theorem 4.11, page 164]).
} 
Note again that $f$ is extended to $\mathbb{R}^{d}$ by being zero outside $S$.

Proof For $(1) \Rightarrow(2)$, the countability of $\mathcal{G}$ makes certain that the argument given in Yosida [25, page 275ff.] essentially goes through in ZF.

For $(2) \Rightarrow(1)$, we use our modified version of the Arzelà-Ascoli theorem (Theorem 2.2.1). To do so, we assimilate elements of the standard proof given for instance in Brezis [6, Proof of Theorem 4.26, page 111ff.], but transform the argument to an argument of more 'sequential' flavour. In our argument, we will use the functions $\rho_{n}$ that for $n \in \mathbb{N}$ are given by

$$
\rho_{n}: \mathbb{R}^{d} \rightarrow \mathbb{R}, \quad \rho_{n}(x):= \begin{cases}\frac{n^{d} e^{\frac{1}{1-\|n x\|^{2}}}}{\int_{B_{1}(0)} e^{\frac{1}{1-\|y\|^{2}}} d y} & \|x\|<1 \\ 0 & \text { else. }\end{cases}
$$

These functions satisfy $\rho_{n} \in \mathcal{C}^{\infty}\left(\mathbb{R}^{d}\right)$, supp $\rho_{n} \subseteq \overline{B_{1 / n}(0)}$ and $\int_{B_{1 / n}(0)} \rho_{n}(x) d x=1$ (see for instance Brezis [6, page 108]).

Now let $\left(f_{n}\right)_{n \in \mathbb{N}}$ be a sequence in $\mathcal{F}$. Then $\mathcal{G}=\left\{f_{n} \mid n \in \mathbb{N}\right\}$ is a countable subset of $\mathcal{F}$. For $n, m \in \mathbb{N}$ define

$$
h_{n, m}:=f_{n} * \rho_{m}
$$

Then define for $m \in \mathbb{N}$

$$
\mathcal{H}_{m}:=\left\{h_{n, m} \mid n \in \mathbb{N}\right\} .
$$

We claim that for each $m \in \mathbb{N}$ we have that $\mathcal{H}_{m}$ is uniformly bounded and equicontinuous. Indeed, uniform boundedness follows from

$$
\left|h_{n, m}(x)\right| \leq \int_{\mathbb{R}^{d}}\left|f_{n}(y)\left\|\rho_{m}(x-y) \mid d y \stackrel{\text { Hölder's inequality }}{\leq}\right\| f_{n}\left\|_{p}\right\| \rho_{m} \|_{p^{\prime}}\right.
$$

where $p^{\prime}$ is the Hölder conjugate of $p$, and similarly equicontinuity follows from

$$
\left|\partial_{x_{j}} h_{n, m}(x)\right| \leq \int_{\mathbb{R}^{d}}\left|f_{n}(y)\right|\left|\partial_{x_{j}} \rho_{m}(x-y)\right| d y \stackrel{\text { Hölder's inequality }}{\leq}\left\|f_{n}\right\|_{p}\left\|\partial_{x_{j}} \rho_{m}\right\|_{p^{\prime}} .
$$

Furthermore, for $m, n \in \mathbb{N}$ we have $\operatorname{supp} h_{n, m} \subseteq \overline{S+B_{1}(0)}$ and $S$ is bounded, which is why our modified Arzelà-Ascoli theorem applies to each $\mathcal{H}_{m}$ and also to all subsets of $\mathcal{H}_{m}$. Now define a function

$$
k: \mathbb{N} \times \mathbb{N} \rightarrow \mathbb{N}
$$

by: $k(n, 1)$ is such that $\left(h_{k(n, 1), 1}\right)_{n \in \mathbb{N}}$ is the convergent subsequence of $\mathcal{H}_{1}$ as given by our modified Arzelà-Ascoli theorem, and if $k(n, m-1)$ is already defined, $k(n, m)$ is 
such that $\left(h_{k(n, m), m}\right)_{n \in \mathbb{N}}$ is the convergent subsequence of $\left(h_{k(n, m-1), m}\right)_{n \in \mathbb{N}} \subseteq \mathcal{H}_{m}$ as given by our modified Arzelà-Ascoli theorem. From this, we define a subsequence $\left(g_{n}\right)_{n \in \mathbb{N}}$ of $\left(f_{n}\right)_{n \in \mathbb{N}}$ by

$$
g_{n}:=f_{k(n, n)} .
$$

We claim that $\left(g_{n}\right)_{n \in \mathbb{N}}$ is a Cauchy sequence in $L^{p}(S)$. Indeed, let $\epsilon>0$. If we set

$$
C_{1}:=\left(\int_{B_{1}(0)}\left|\rho_{1}(y)\right|^{p^{\prime}} d y\right)^{p / p^{\prime}} \text { and } C_{2}:=\int_{B_{1}(0)} 1 d y
$$

we get for all $g \in \mathcal{G}$

$$
\begin{aligned}
\int_{S}\left|g * \rho_{n}(x)-g(x)\right|^{p} d x & =\int_{S}\left|\int_{B_{1 / n}(0)} \rho_{n}(y) g(x-y) d y-\int_{B_{1 / n}(0)} \rho_{n}(y) g(x) d y\right|^{p} d x \\
& \leq \int_{S}\left(\int_{B_{1}(0)}\left|\rho_{1}(y) \| g(x-y / n)-g(x)\right| d y\right)^{p} d x \\
& \stackrel{\leq}{\leq} C_{1} \int_{S} \int_{B_{1}(0)}|g(x-y / n)-g(x)|^{p} d y d x \\
& \stackrel{\text { Fubini's theorem }}{=} C_{1} \int_{B_{1}(0)} \int_{S}|g(x-y / n)-g(x)|^{p} d x d y \\
& \leq C_{1} C_{2} \sup _{\|y\|<1} \int_{S}|g(x-y / n)-g(x)|^{p} d x
\end{aligned}
$$

and hence, taking the supremum over $g \in \mathcal{G}$ of this, first on the right hand side and then on the left hand side, we get

$$
\sup _{g \in \mathcal{G}} \int_{S}\left|g * \rho_{n}(x)-g(x)\right|^{p} d x \leq C_{1} C_{2} \sup _{g \in \mathcal{G}} \sup _{\|y\|<1} \int_{S}|g(x-y / n)-g(x)|^{p} d x .
$$

Therefore, by our assumption on countable subsets of $\mathcal{F}$, we may choose $J \in \mathbb{N}$ sufficiently large so that for all $g \in \mathcal{G}$

$$
\left\|g * \rho_{J}-g\right\|_{p} \leq \epsilon / 3 .
$$

Furthermore, by construction, the sequence $\left(h_{k(J, n), J}\right)_{n \in \mathbb{N}}$ is a Cauchy sequence in $\mathcal{C}\left(\overline{S+B_{1}(0)}\right)$ and hence also in $L^{p}(S)$, since $S$ is bounded. Hence we may pick $M \in \mathbb{N}$ such that for all $n, m \geq M$ we have

$$
\left\|h_{k(n, J), J}-h_{k(m, J), J}\right\|_{p}<\epsilon / 3 .
$$

Then set $N:=\max \{J, M\}$ to obtain for $m, n \geq N$ that

$$
\begin{aligned}
\left\|g_{n}-g_{m}\right\|_{p} & \leq\left\|g_{n}-h_{k(n, n), J}\right\|_{p}+\left\|h_{k(n, n), J}-h_{k(m, m), J}\right\|_{p}+\left\|h_{k(m, m), J}-g_{m}\right\|_{p} \\
& <\epsilon / 3+\epsilon / 3+\epsilon / 3=\epsilon .
\end{aligned}
$$




\subsection{The relationship to the axiom of choice}

We first note that the cardinality of separable metric spaces is always less than or equal to the cardinality of the real numbers $\mathbb{R}$ (every separable metric space is homeomorphic to a subspace of the Hilbert cube, see for instance Bourbaki [3, Proposition 12, page 156]). Then we note that for a compact $K \subset \mathbb{R}^{d}$, the space $\mathcal{C}(K)$ of continuous, real valued functions with domain of definition $K$ is separable (this may be proven without the axiom of choice by approximating any function in such a space by a suitable multi-dimensional Bernstein polynomial - see for instance Reimer [17, Section 5.2, page 119ff.] — and then in turn approximating the Bernstein polynomial by a rational polynomial, where the set of rational polynomials is countable). Further we note that for any measurable set $S \subseteq \mathbb{R}^{d}$, the space $L^{p}(S)$ may be regarded as a subspace of $L^{p}\left(\mathbb{R}^{d}\right)$ by identifying equivalence classes from $L^{p}(S)$ with equivalence classes of $L^{p}\left(\mathbb{R}^{d}\right)$ which are almost everywhere zero on $\mathbb{R}^{d} \backslash S$ through the obvious bijective function, which preserves metric. Now the space $L^{p}\left(\mathbb{R}^{d}\right)$ is separable (see for instance Brezis [6, Theorem 4.13, page $98 \mathrm{ff}]$.$) . Thus, we conclude that the cardinality of$ both $\mathcal{C}(K)$ and $L^{p}(S)$ are less than or equal to the cardinality of the real numbers $\mathbb{R}$. Furthermore, the functions $\mathbb{R} \ni x \mapsto x \mathbf{1}_{K} \in \mathcal{C}(K)(K \neq \emptyset)$ and $\mathbb{R} \ni x \mapsto\left[x \mathbf{1}_{S}\right] \in L^{p}(S)$ ( $S \subseteq \mathbb{R}^{d}$ with nonzero measure, square brackets indicating equivalence class formation) are injections, which is why the cardinalities of $\mathcal{C}(K)(K \neq \emptyset)$ and $L^{p}(S)\left(S \subseteq \mathbb{R}^{d}\right.$ with nonzero measure) are equal to the cardinality of $\mathbb{R}$ by the Schröder-Bernstein theorem (for the statement and a choiceless proof of the Schröder-Bernstein theorem see for instance Halmos [9, Chapter 22, page $88 \mathrm{ff}$.]). With this in mind, we now explicate the relationship between the axiom of choice and the Arzelà-Ascoli and Fréchet-Kolmogorov theorems.

Our investigation uses the same method deployed in Rhineghost [18] and is thus based on the following result proved by Herrlich and Strecker [13, Main Theorem, page 553]:

2.3.1 Theorem The axiom of countable choice for subsets of real numbers is equivalent to the statement 'Every unbounded subset of $\mathbb{R}$ contains a countable, unbounded subset.'

Furthermore, we use the following result (see for instance Hušek [14, Remark on page 290]):

2.3.2 Theorem Given a bounded, equicontinuous set of functions $\mathcal{F} \subseteq \mathcal{C}(K)$ where $K \subset \mathbb{R}^{d}$ is compact, one may extend each function in $\mathcal{F}$ to produce a bounded, equicontinuous set of functions defined on $\overline{B_{R}(0)}$, where $R$ is such that $K \subseteq \overline{B_{R}(0)}$. 
2.3.3 Theorem Assume the validity of ZF. Then the Arzelà-Ascoli theorem (Theorem 2.1.1) is true if and only if the axiom of countable choice for subsets of the real numbers $\mathbb{R}$ is true.

Proof We first prove the Arzelà-Ascoli theorem from the axiom of countable choice. Indeed, $(2) \Rightarrow(1)$ in Theorem 2.1.1 is covered by Theorem 2.2.1. For $(1) \Rightarrow(2)$, we argue by contradiction: When $\mathcal{F}$ is not bounded, we use the axiom of countable choice for subsets of the real numbers to obtain an unbounded countable set $\mathcal{G} \subseteq \mathcal{F}$; when $\mathcal{F}$ is not equicontinuous, there exists an $\epsilon>0$ and an $x \in K$ such that

$$
S_{n}:=\left\{f \in \mathcal{F}\left|\exists y \in B_{1 / n}(x):\right| f(x)-f(y) \mid \geq \epsilon\right\}
$$

is nonempty for every $n>0$, and we apply the axiom of countable choice for subsets of the reals to get a countable, non-equicontinuous $\mathcal{G} \subset \mathcal{F}$. In both cases, first we extend everything to a suitable $\overline{B_{R}(0)}$ using Theorem 2.3.2 to ensure separability of the domain of definition of the set of functions, and then apply Theorem 2.2.1 to obtain a contradiction.

Then we deduce the axiom of countable choice from the Arzelà-Ascoli Theorem 2.1.1. Indeed, let $S \subseteq \mathbb{R}$ be an unbounded subset of $\mathbb{R}$, and set $K:=\overline{B_{R}(0)}$, where $R>0$. Set

$$
\mathcal{F}:=\{x \mapsto s \mid s \in S\} \subset \mathcal{C}(K),
$$

the constant functions for the elements of $S$. By the Arzelà-Ascoli theorem, $\mathcal{F}$ contains a sequence which does not have a convergent subsequence, and hence by our modified Arzelà-Ascoli Theorem 2.2.1, there exists a countable $\mathcal{G} \subseteq \mathcal{F}$ that is either not bounded or not equicontinuous. But $\mathcal{F}$ is equicontinuous, hence $\mathcal{G}$ is unbounded. The theorem follows from Theorem 2.3.1.

2.3.4 Theorem Assume the validity of $Z F$. Then the Fréchet-Kolmogorov theorem is true if and only if the axiom of countable choice for subsets of the real numbers $\mathbb{R}$ is true.

Proof We first prove the Fréchet-Kolmogorov theorem from the axiom of countable choice for subsets of the real numbers. Indeed, $(2) \Rightarrow(1)$ is covered by Theorem 2.2.2, and for $(1) \Rightarrow(2)$ we suppose for a contradiction that either $\mathcal{F}$ is unbounded or that $\mathcal{F}$ does not satisfy

$$
\lim _{h \rightarrow 0} \sup _{f \in \mathcal{F}} \int_{S}|f(x+h)-f(x)|^{p} d x=0 .
$$


If $\mathcal{F}$ is unbounded, we may pick an unbounded countable subset $\mathcal{G} \subseteq \mathcal{F}$ (apply countable choice for subsets of the reals to $\left\{f \in \mathcal{F} \mid\|f\|_{p}>n\right\}$ ), and if $\mathcal{F}$ does not satisfy equation (a), then there exists $\epsilon>0$ such that for all $n$, the set

$$
S_{n}:=\left\{f \in \mathcal{F}\left|\exists h \in B_{1 / n}(0): \int_{S}\right| f(x+h)-\left.f(x)\right|^{p} d x>\epsilon\right\}
$$

is nonempty, and by choosing from these sets we get a countable $\mathcal{G} \subseteq \mathcal{F}$ that does not satisfy (a) with $f \in \mathcal{G}$. In both cases Theorem 2.2.2 gives a contradiction.

To prove that the axiom of countable choice for subsets of real numbers follows from the Fréchet-Kolmogorov theorem, we proceed exactly as in the proof of Theorem 2.3.3.

\section{The uniform boundedness principle}

\subsection{Introduction}

The uniform boundedness principle may be stated as follows (see for instance Brezis [6, Theorem 2.2, page 32]):

3.1.1 Theorem (Uniform boundedness principle) Let $\left(X,\|\cdot\|_{X}\right)$ be a Banach space, $\left(Y,\|\cdot\|_{Y}\right)$ a normed space. Let $\left(T_{\alpha}\right)_{\alpha \in A}$ be a family of linear and continuous functions from $X$ to $Y$. If

$$
\forall x \in X: \sup _{\alpha \in A}\left\|T_{\alpha}(x)\right\|_{Y}<\infty
$$

then

$$
\sup _{\alpha \in A}\left\|T_{\alpha}\right\|_{o p}<\infty
$$

Roughly speaking, this theorem could be described as asserting: 'If a family of linear and continuous functions is pointwise bounded, it is also bounded with regard to the operator norm.'

In what follows, we will prove that the axiom of countable choice implies the uniform boundedness principle and that several axioms follow from the uniform boundedness principle. In fact, this will also clarify the choice-axiomatic strength of several other theorems. This is due to the following:

3.1.2 Theorem The following are equivalent in $Z F$ : 
(1) Every Banach space is barrelled.

(2) Every lower semi-continuous seminorm on a Banach space is continuous.

(3) The uniform boundedness principle holds.

Proof $(1) \Leftrightarrow(2) \quad$ Schechter [21, 27.32 and 27.33, page 737]

(2) $\Rightarrow$ (3) Bourbaki [5, Theorem 1, page III.25] and Bourbaki [4, Theorem 4, page 362]

(3) $\Rightarrow$ (1) Schechter [21, 27.35, page 738ff.]

3.1.3 Theorem The following are equivalent in $Z F$ :

(1) The closed graph theorem (ie, if $X, Y$ are Banach spaces and $T: X \rightarrow Y$ is a linear function such that

$$
\operatorname{graph} T:=\{(x, T(x)) \mid x \in X\} \subset X \times Y
$$

is closed, then $T$ is continuous) holds.

(2) A sequential version of the closed graph theorem (ie, if $X, Y$ are Banach spaces and $T: X \rightarrow Y$ is a linear function such that

$$
\operatorname{graph} T:=\{(x, T(x)) \mid x \in X\} \subset X \times Y
$$

is sequentially closed ${ }^{3}$, then $T$ is continuous) holds.

(3) The open mapping theorem (ie, whenever $X$ and $Y$ are Banach spaces and $T: X \rightarrow Y$ is a linear, continuous and surjective function, then $T$ is open) holds.

Proof $(3) \Rightarrow(2) \quad$ Brezis [6, Theorem 2.9, page 37 and Corollaries 7 and 8, page 35] $(1) \Rightarrow(3)$ Robertson and Robertson [19, Theorem 3, page 12]

Further, from Schechter [21, 27.34, page 737ff.] it follows that any of the statements listed in 3.1.3 implies the statements in 3.1.2.

Our knowledge apart from this article of the relation between the uniform boundedness principle and the axiom of choice stems from an article by Norbert Brunner [7]. Indeed, he proved that if every Banach space is barrelled, then the axiom of countable multiple

\footnotetext{
${ }^{3}$ Note that the statement 'In every metric space, a set is closed if and only it is sequentially closed' is equivalent to the axiom of countable choice (see [8, Theorem 2.1, page 146]); however, closed sets are always sequentially closed, and for graphs the equivalence holds in $\mathrm{ZF}$, as the theorem shows. In particular, for linear functions between Banach spaces, continuity and sequential continuity are equivalent in $\mathrm{ZF}$.

${ }^{4}$ Note that when $\tau: X \times Y \rightarrow X \times Y, \tau(x, y):=(x, y-T x)$, then $\operatorname{graph} T=\tau^{-1}(X \times\{0\})$.
} 
choice (see for instance Herrlich [12, Definition 2.10, page 14] or indeed Axiom 3.3.2) holds (see Brunner [7, Lemma 4, page 124ff.]). Furthermore, he proved that given ZF and the axiom of countable finite choice (ie, from a sequence of finite sets one can extract a sequence of members), the axiom of countable multiple choice suffices to prove that every Banach space is barrelled (see Brunner [7, proof of Lemma 5, page 125ff.]).

Combining this with Theorem 3.1.2 and the fact that trivially, the axiom of countable choice implies both the axiom of countable multiple choice and the axiom of countable finite choice, it follows that

(1) the axiom of countable choice implies the uniform boundedness principle, and

(2) the uniform boundedness principle implies the axiom of countable multiple choice.

In what follows, we will give new, direct proofs of these two facts, and further deduce two additional choice-like axioms from the uniform boundedness principle.

\subsection{Countable choice implies the uniform boundedness principle}

In slightly modifying an argument given by Alan D. Sokal [22], we are able to prove the uniform boundedness principle using nothing more than the Zermelo-Fraenkel system and the axiom of countable choice (as noted above, this has been done before).

3.2.1 Theorem Assume the Zermelo-Fraenkel system and the axiom of countable choice. Then the uniform boundedness principle holds.

Proof Assume for a contradiction that $\left(T_{\alpha}\right)_{\alpha \in A}$ is an unbounded family of linear, continuous functions from a Banach space $\left(X,\|\cdot\|_{X}\right)$ to a Banach space $\left(Y,\|\cdot\|_{Y}\right)$. Then all the sets

$$
A_{n}:=\left\{T_{\alpha} \mid \alpha \in A,\left\|T_{\alpha}\right\|_{o p}>4^{n}\right\}
$$

are nonempty. Hence, by the axiom of countable choice, we may pick a sequence $\left(T_{n}\right)_{n \in \mathbb{N}}$ such that for each $n \in \mathbb{N}$ we have $T_{n} \in A_{n}$. By definition of the operator norm, all the sets

$$
B_{n}:=\left\{x \in X \mid\|x\|_{X} \leq 1,\left\|T_{n}(x)\right\|_{Y}>\frac{2}{3}\left\|T_{n}\right\|_{o p}\right\}
$$

are nonempty. A second application of the axiom of countable choice hence permits us to choose a sequence $\left(x_{n}\right)_{n \in \mathbb{N}}$ such that for all $n \in \mathbb{N}$, we have $x_{n} \in B_{n}$. 
Now define a function $f: \mathbb{N} \times X \rightarrow X$ by

$$
f(n, x):= \begin{cases}x+3^{-(n+1)} x_{n+1} & \left\|T_{n+1}\left(x+3^{-(n+1)} x_{n+1}\right)\right\|_{Y}>3^{-(n+1)} \frac{2}{3}\left\|T_{n+1}\right\|_{o p} \\ x-3^{-(n+1)} x_{n+1} & \text { else. }\end{cases}
$$

We claim that for each $n \in \mathbb{N}$ there exists exactly one $n$-tuple $\left(z_{n, 1}, \ldots, z_{n, n}\right)$ such that

(1) $z_{n, 1}=x_{1}$

(2) $z_{n, k+1}=f\left(k, z_{n, k}\right)$ for $k \in\{1, \ldots, n-1\}$.

Existence is proved by induction on $n$; for uniqueness, assume otherwise and use that $f$ is a function and hence can have only one value. For a given $n$, we have by induction on $k$ that $z_{n, k}=z_{n-1, k}$ for $k \in\{1, \ldots, n-1\}$. Define a sequence $\left(y_{n}\right)_{n \in \mathbb{N}}$ by $y_{n}:=z_{n, n}$. We get for $n \in \mathbb{N}$

$$
y_{n+1}=z_{n+1, n+1}=f\left(n, z_{n+1, n}\right)=f\left(n, z_{n, n}\right)=f\left(n, y_{n}\right) .
$$

The sequence $\left(y_{n}\right)_{n \in \mathbb{N}}$ has two properties:

(1) When $k, n \in \mathbb{N}$, we have

$$
\left\|y_{n}-y_{n+k}\right\| \leq \sum_{j=0}^{\infty}\left\|y_{n+j+1}-y_{n+j}\right\| \leq \sum_{j=0}^{\infty} 3^{-(n+j+1)}=3^{-(n+1)} \frac{3}{2}=3^{-n} \frac{1}{2} .
$$

(2) For $x \in X$ (since the maximum is larger than the average and due to the triangle inequality)

$$
\begin{aligned}
\max & \left\{\left\|T_{n}\left(x+3^{-n} x_{n}\right)\right\|,\left\|T_{n}\left(x-3^{-n} x_{n}\right)\right\|\right\} \\
& \geq \frac{1}{2}\left(\left\|T_{n}\left(x+3^{-n} x_{n}\right)\right\|+\left\|T_{n}\left(x-3^{-n} x_{n}\right)\right\|\right) \geq 3^{-n}\left\|T_{n}\left(x_{n}\right)\right\| \geq 3^{-n} \frac{2}{3}\left\|T_{n}\right\|_{o p}
\end{aligned}
$$

and hence $\quad\left\|T_{n}\left(y_{n}\right)\right\| \geq 3^{-n} \frac{2}{3}\left\|T_{n}\right\|_{o p}$.

From the first property, $\left(y_{n}\right)_{n \in \mathbb{N}}$ is a Cauchy sequence, hence convergent to some $y \in X$. Then for $k, n \in \mathbb{N}$

$$
\left\|y_{n}-y\right\| \leq\left\|y_{n}-y_{k}\right\|+\left\|y_{k}-y\right\|,
$$

and letting $k \rightarrow \infty$ proves, together with the first property, that $\left\|y_{n}-y\right\| \leq 3^{-n} / 2$.

Combining this with the second property, we get

$$
\begin{aligned}
\left\|T_{n}(y)\right\| & \geq\left\|T_{n}\left(y_{n}\right)\right\|-\left\|T_{n}\left(y_{n}-y\right)\right\| \geq 3^{-n} \frac{2}{3}\left\|T_{n}\right\|_{o p}-3^{-n} \frac{1}{2}\left\|T_{n}\right\|_{o p} \\
& =\frac{1}{6} 3^{-n}\left\|T_{n}\right\|_{o p}>\frac{1}{6}(4 / 3)^{n} \rightarrow \infty
\end{aligned}
$$

that is, $\left(T_{\alpha}\right)_{\alpha \in A}$ is unbounded in $y$. 
Note that instead of using the axiom of countable choice in selecting the functions $\left(T_{n}\right)_{n \in \mathbb{N}}$, we could have instead defined the $B_{n}$ as

$$
B_{n}:=\left\{x \in X \mid\|x\|_{X} \leq 1, \exists \alpha \in A:\left\|T_{\alpha}(x)\right\|_{Y}>\frac{2}{3}\left\|T_{\alpha}\right\|_{o p}>\frac{2}{3} 4^{n}\right\}
$$

and only used the axiom of countable choice on the $B_{n}$. Then we would have replaced all the inequalities by inequalities for which there exists a suitable $\alpha$ such that they hold.

\subsection{Axioms implied by the uniform boundedness principle}

\subsubsection{General proof strategy}

Clearly, as became apparent in the proof of the last subsection, the uniform boundedness principle may be reformulated as follows:

3.3.1 Theorem (Uniform boundedness principle, reformulated) Let $\left(T_{\alpha}\right)_{\alpha \in A}$ be an unbounded family of linear functions from a Banach space $X$ to a normed space $Y$. Then there exists $x \in X$ such that $\left\{T_{\alpha}(x) \mid \alpha \in A\right\} \subseteq Y$ is unbounded.

Hence, we see that given an unbounded family of linear, continuous functions, the uniform boundedness principle translates into an existence claim, namely the existence of a point where the respective family of linear, continuous functions is unbounded. If we therefore are able to associate to such a point subsets of a given family of sets, we can use an existence result as such to obtain variants of the axiom of choice. In fact, using the right Banach spaces, this will be possible. The spaces considered in this paper are created using the following construction:

Assume we are given a countable family of Banach spaces $X_{1}, X_{2}, \ldots, X_{n}, \ldots$. Then we may construct from them new Banach spaces in which $X_{1}, X_{2}, \ldots, X_{n}, \ldots$ are isometrically contained in a canonical fashion. Namely, if $1 \leq p \leq \infty$, set

$$
\bigoplus_{n \in \mathbb{N}}^{p} X_{n}:=\left\{\left(x_{n}\right)_{n \in \mathbb{N}} \in \prod_{n \in \mathbb{N}} X_{n} \mid\left\|\left(x_{n}\right)_{n \in \mathbb{N}}\right\|_{p}<\infty\right\}
$$

where the norm $\|\cdot\|_{p}$ is given by

$$
\left\|\left(x_{n}\right)_{n \in \mathbb{N}}\right\|_{p}:= \begin{cases}\left(\sum_{n=1}^{\infty}\left\|x_{n}\right\|_{X_{n}}^{p}\right)^{1 / p} & p<\infty \\ \sup _{n \in \mathbb{N}}\left\|x_{n}\right\|_{X_{n}} & p=\infty\end{cases}
$$


where for each $n\|\cdot\|_{X_{n}}$ is the norm of $X_{n}$. All these spaces are Banach spaces (see for instance Helemskii [10, page 127]). The space

$$
\bigoplus_{n \in \mathbb{N}}^{p} X_{n}
$$

is called the $\ell^{p}$ sum of $X_{1}, X_{2}, \ldots, X_{n}, \ldots$

In what follows, we will associate to a sequence of sets $\left(S_{n}\right)_{n \in \mathbb{N}}$ spaces $\left(X_{n}\right)_{n \in \mathbb{N}}$. The choice axioms that we want to deduce from the uniform boundedness principle will assert that given a sequence $\left(S_{n}\right)_{n \in \mathbb{N}}$, for infinitely many $n$ we can choose sets $M_{n} \subseteq S_{n}$ whose cardinality obeys a certain restriction (for instance is finite or obeys some bound). In order to execute the deduction, we pick the spaces $\left(X_{n}\right)_{n \in \mathbb{N}}$ such that, for a given $n$, all elements of $X_{n}$ having a certain property (for instance, nonzero elements) yield a set $M_{n}$ obeying the desired condition; this is the case when the elements of $X_{n}$ with the certain property are all sufficiently 'asymmetric' in their structure. Then we use the uniform boundedness principle, applied to a suitable unbounded family of linear, continuous functions (which will be defined on a suitable $\ell^{p}$ sum of the $\left(X_{n}\right)_{n \in \mathbb{N}}$ ), to get elements of $X_{n}$ for infinitely many $n$ which have the certain property that will yield a suitable $M_{n}$.

In our exposition, we will dedicate a subsubsection to each axiom that will be deduced from the uniform boundedness principle. In each subsubsection, we will start with a lemma explaining why the elements with the certain property are sufficiently asymmetric such that subsets of the desired cardinality may be selected.

\subsubsection{Choosing finite subsets}

We will now present another proof for the fact that the uniform boundedness principle implies the axiom of countable multiple choice, which is defined as follows (see for instance [12, Definition 2.10, page 14]):

3.3.2 Axiom (Countable multiple choice) Let $\left(S_{n}\right)_{n \in \mathbb{N}}$ be a sequence of sets. Then there exists a sequence of nonempty sets $\left(M_{n}\right)_{n \in \mathbb{N}}$ such that for all $n$ we have $M_{n} \subseteq S_{n}$ and $M_{n}$ is finite.

We will use the fact that this axiom is equivalent to the following seemingly weaker axiom:

3.3.3 Axiom (Partial countable multiple choice) Let $\left(S_{n}\right)_{n \in \mathbb{N}}$ be a sequence of sets. Then there exists an infinite $I \subseteq \mathbb{N}$ and a family of nonempty sets $\left(M_{n}\right)_{n \in I}$ such that for all $n \in I$ we have $M_{n} \subseteq S_{n}$ and $M_{n}$ is finite. 
The proof of equivalence of the two axioms is essentially the same as the proof of equivalence of the axiom of partial countable choice to the axiom of countable choice (see for instance Herrlich [12, Theorem 2.12 3., page 15]) and follows easily from Keremedis [16, Lemma 1.2, page 570].

Let $S$ be an arbitrary set. $\mathcal{P}(S)$, the power set of $S$, is a $\sigma$-algebra, and the counting measure on $S$ is defined as

$$
\mu(E)= \begin{cases}\# E & \# E<\infty \\ \infty & \text { otherwise }\end{cases}
$$

where $E \subseteq S$ is arbitrary (see for instance Tao [24, Example 1.4.26, page 90ff.]). $L^{1}(S, \mu)$ is a Banach space (see for instance Brezis [6, Theorem 4.8, page 93]).

We note the following lemma regarding $L^{1}(S, \mu)$ :

3.3.4 Lemma Let $\left(S_{\alpha}\right)_{\alpha \in A}$ be a family of nonempty sets, and let $\mu_{\alpha}$ be the counting measure on $S_{\alpha}$. There exists a function

$\Phi_{\left(S_{\alpha}\right)_{\alpha \in A}}: \bigcup_{\alpha \in A}\left\{f \in L^{1}\left(S_{\alpha}, \mu_{\alpha}\right) \mid \int_{S_{\alpha}} f(\sigma) d \mu_{\alpha}(\sigma) \neq 0\right\} \rightarrow \bigcup_{\alpha \in A}\left\{T \subseteq S_{\alpha} \mid 0<\# T<\infty\right\}$

such that whenever $f \in L^{1}\left(S_{\alpha}, \mu_{\alpha}\right)$, then $\Phi_{\left(S_{\alpha}\right)_{\alpha \in A}}(f) \subseteq S_{\alpha}$.

Proof Let $\alpha \in A$ and $f \in L^{1}\left(S_{\alpha}, \mu_{\alpha}\right)$ such that $\int_{S_{\alpha}} f(\sigma) d \mu_{\alpha}(\sigma) \neq 0$ be given. We partition the split real number line $\mathbb{R} \backslash\{0\}$ into countably many subsets as thus:

$$
\mathbb{R} \backslash\{0\}=\bigcup_{n \in \mathbb{Z}}\left(\left[-2^{n+1},-2^{n}\right) \cup\left(2^{n}, 2^{n+1}\right]\right) .
$$

The following three observations are immediate:

(1) For all $n \in \mathbb{Z}$, at most finitely many elements of $f\left(S_{\alpha}\right)$ are in $\left[-2^{n+1},-2^{n}\right) \cup$ $\left(2^{n}, 2^{n+1}\right]$.

(2) $\left[-2^{n+1},-2^{n}\right) \cup\left(2^{n}, 2^{n+1}\right]$ contains a point of $f\left(S_{\alpha}\right)$ only for finitely many positive $n$.

(3) $\mathbb{R} \backslash\{0\}$, and hence at least one of the sets $\left[-2^{n+1},-2^{n}\right) \cup\left(2^{n}, 2^{n+1}\right]$, must contain a point of $f\left(S_{\alpha}\right)$.

Hence, we choose $n \in \mathbb{Z}$ maximal such that there are some points of $f\left(S_{\alpha}\right)$ in $\left[-2^{n+1},-2^{n}\right) \cup\left(2^{n}, 2^{n+1}\right]$; these are finitely many, and we define $\Phi_{\left(S_{\alpha}\right)_{\alpha} \in A}(f)$ to be the set of the preimages of these points. 
3.3.5 Theorem Assume that the uniform boundedness principle holds. Then, given a sequence $\left(S_{n}\right)_{n \in \mathbb{N}}$ of nonempty sets, there exists a sequence of sets $\left(M_{n}\right)_{n \in \mathbb{N}}$ such that for all $n \in \mathbb{N} M_{n} \subseteq S_{n}$ and $\# M_{n}<\infty$ and for infinitely many $n, M_{n} \neq \emptyset$.

Proof For each $n \in \mathbb{N}$, set $X_{n}:=L^{1}\left(S_{n}, \mu_{n}\right)$, where $\mu_{n}$ is the counting measure on $S_{n}$. Then set

$$
X:=\bigoplus_{n \in \mathbb{N}}^{p} X_{n}
$$

where the choice of $1 \leq p \leq \infty$ does not matter; we may for instance take $p=1$. Set $Y:=\mathbb{R}$, where the norm is given by the absolute value of the reals. For each $n$ define a linear function $T_{n}: X \rightarrow Y$ by

$$
T_{n}\left(\left(x_{n}\right)_{n \in \mathbb{N}}\right):=4^{n} \int_{S_{n}} x_{n}(\sigma) d \mu_{n}(\sigma) .
$$

If, for a fixed $n$, a $\sigma \in S_{n}$ is picked, and the element $x=\left(x_{k}\right)_{k \in \mathbb{N}} \in X$ defined by the sequence

$$
x_{k}= \begin{cases}0 & n \neq k \\ \mathbf{1}_{\{\sigma\}} & n=k\end{cases}
$$

is considered (where $\mathbf{1}_{A}$ is defined to be the indicator function on the set $A$ ), it becomes evident that $\left\|T_{n}\right\|_{o p} \geq 4^{n}$; that is, the family of linear functions $\left(T_{n}\right)_{n \in \mathbb{N}}$ is uniformly unbounded. Hence, by the uniform boundedness principle, it is also pointwise unbounded. In particular, we find $x=\left(x_{n}\right)_{n \in \mathbb{N}} \in X$ such that

$$
\int_{S_{n}} x_{n}(\sigma) d \mu_{n}(\sigma) \neq 0
$$

for infinitely many $n$, say for all $n \in I$, where $I \subseteq \mathbb{N}$ is infinite. For all such $n$, we then define $M_{n}:=\Phi_{\left(S_{n}\right)_{n \in I}}\left(x_{n}\right)$, where $\Phi_{\left(S_{n}\right)_{n \in I}}$ is as in Lemma 3.3.4.

Hence, the uniform boundedness principle implies the axiom of partial countable multiple choice, and thus the axiom of countable multiple choice.

\subsubsection{Choosing subsets of asymptotically bounded cardinality}

Now I will deduce from the uniform boundedness principle the following choice axiom:

3.3.6 Axiom (Axiom of partial countable asymptotic choice) Let $\left(S_{n}\right)_{n \in \mathbb{N}}$ be a sequence of finite nonempty sets, and let $\left(\lambda_{n}\right)_{n \in \mathbb{N}}$ be a sequence of positive real numbers which is unbounded. Then there exists an infinite set $I \subseteq \mathbb{N}$, a family of sets $\left(M_{n}\right)_{n \in I}$ and a constant $C>0$ such that for all $n \in I$ we have $M_{n} \subseteq S_{n}, M_{n} \neq \emptyset$ and $\# M_{n} \leq C \lambda_{n}$. 
The deduction of this axiom is based on the following lemma:

3.3.7 Lemma Let $S$ be a finite set, $\mu$ the counting measure on $S$, and $f: S \rightarrow \mathbb{R}$ a real-valued function on $S$. If for a $C>0$ we have

$$
\int_{S}|f(\sigma)| d \mu(\sigma) \leq C \sup _{\sigma \in S}|f(\sigma)|
$$

then \# $\left\{\sigma \in S|| f(\sigma)\left|=\sup _{\sigma \in S}\right| f(\sigma) \mid\right\} \leq C$.

Proof Assume otherwise. Then

$$
\int_{S}|f(\sigma)| d \mu(\sigma) \geq \#\left\{\sigma \in S|f(\sigma)|=\sup _{\sigma \in S}|f(\sigma)|\right\} \cdot \sup _{\sigma \in S}|f(\sigma)|>C \sup _{\sigma \in S}|f(\sigma)| .
$$

3.3.8 Theorem The uniform boundedness principle implies Axiom 3.3.6.

Proof Let $\left(S_{n}\right)_{n \in \mathbb{N}}$ be a sequence of finite, nonempty sets, and let $\left(\lambda_{n}\right)_{n \in \mathbb{N}}$ be an unbounded sequence of positive, real numbers. Let $\mu_{n}$ be the counting measure on $S_{n}$. Set $X_{n}:=L^{1}\left(S_{n}, \mu_{n}\right)$ and $Y_{n}:=L^{\infty}\left(S_{n}, \mu_{n}\right)$ and then

$$
X:=\bigoplus_{n \in \mathbb{N}}^{\infty} X_{n} \text { and } Y:=\bigoplus_{n \in \mathbb{N}}^{p} Y_{n}
$$

The choice of $1 \leq p \leq \infty$ doesn't matter, eg $p=1$. Define a sequence of linear functions $T_{n}: X \rightarrow Y$ by

$$
T_{n}\left(\left(x_{k}\right)_{k \in \mathbb{N}}\right):=\lambda_{n}\left(\delta_{n k} x_{k}\right)_{k \in \mathbb{N}},
$$

where

$$
\delta_{n k}= \begin{cases}1 & n=k \\ 0 & \text { else }\end{cases}
$$

is the Kronecker delta. If, for a fixed $n$, a $\sigma \in S_{n}$ is picked, and the element $x=\left(x_{k}\right)_{k \in \mathbb{N}} \in X$ defined by the sequence

$$
x_{k}= \begin{cases}0 & n \neq k \\ \mathbf{1}_{\{\sigma\}} & n=k\end{cases}
$$

is considered, it becomes evident that $\left\|T_{n}\right\|_{o p} \geq \lambda_{n}$. It follows that the family of linear functions $\left(T_{n}\right)_{n \in \mathbb{N}}$ is uniformly unbounded, and hence, by the uniform boundedness principle, pointwise unbounded. Hence pick $\left(x_{k}\right)_{k \in \mathbb{N}} \in X$ such that the set $\left\{\left\|T_{n}\left(\left(x_{k}\right)_{k \in \mathbb{N}}\right)\right\|_{Y} \mid n \in \mathbb{N}\right\}$ is an unbounded subset of the real numbers. In particular, there exists an infinite set $I \subseteq \mathbb{N}$ such that for all $n \in I$ we have

$$
1 \leq\left\|T_{n}\left(\left(x_{k}\right)_{k \in \mathbb{N}}\right)\right\|_{Y}=\lambda_{n}\left\|\left(\delta_{n k} x_{k}\right)_{k \in \mathbb{N}}\right\|_{Y}=\lambda_{n} \max _{\sigma \in S_{n}}\left|x_{n}(\sigma)\right|
$$


But since $\left(x_{k}\right)_{k \in \mathbb{N}} \in X$, there exists a $C>0$ such that

$$
\forall n \in \mathbb{N}: C \geq\left\|x_{n}\right\|_{X_{n}}=\int_{S_{n}}\left|x_{n}(\sigma)\right| d \mu_{n}(\sigma) .
$$

Hence, for all $n \in I$ we have

$$
\int_{S_{n}}\left|x_{n}(\sigma)\right| d \mu_{n}(\sigma) \leq C \leq C \lambda_{n} \max _{\sigma \in S_{n}}\left|x_{n}(\sigma)\right| .
$$

By Lemma 3.3.7, by defining $M_{n}:=\left\{\sigma \in S_{n}|| x_{n}(\sigma)\left|=\sup _{\sigma \in S_{n}}\right| x_{n}(\sigma) \mid\right\}$ for all $n \in I$ we get a family of sets as required by Axiom 3.3.6.

\subsubsection{Choosing singletons}

3.3.9 Axiom (Axiom of countable choice for sets of bounded, finite cardinality) The axiom of countable choice for sets of bounded, finite cardinality shall mean the following: If $n \in \mathbb{N}$ and $\left(S_{k}\right)_{k \in \mathbb{N}}$ is a sequence of nonempty sets such that $\forall k \in \mathbb{N}: \# S_{k} \leq n$, then

$$
\prod_{k \in \mathbb{N}} S_{k} \neq 0
$$

3.3.10 Definition Let $S$ be a set of finite cardinality, say $\# S=n \in \mathbb{N}$. Then define

$$
\begin{aligned}
U_{S} & :=\left\{\left(x_{\sigma}\right)_{\sigma \in S} \mid \forall \sigma \in S: x_{\sigma} \in \mathbb{R}\right\} \\
V_{S} & :=\left\{t(1)_{\sigma \in S} \mid t \in \mathbb{R}\right\} \\
W_{S} & :=U_{S} / V_{S}
\end{aligned}
$$

Trivially, the following holds:

3.3.11 Lemma Let $S$ be a set of finite cardinality and $w=\left(x_{\sigma}\right)_{\sigma \in S}+V_{S} \in W_{S}$ such that $w \neq 0$. Then the set

$$
\left\{\sigma \in S \mid x_{\sigma} \neq \min _{\rho \in S} x_{\rho}\right\}
$$

is independent of the representative of $w$ and a nonempty, proper subset of $S$.

3.3.12 Theorem Assume that the uniform boundedness principle is true. Then Axiom 3.3.9 holds.

Proof Let a family of nonempty sets $\left(S_{k}\right)_{k \in \mathbb{N}}$ be given such that for all $k$ we have $\# S_{k} \leq n$. For each $k$, we form the spaces $U_{S_{k}}$ and $W_{S_{k}}$ as given in Definition 3.3.10. These are finite-dimensional real vector spaces and hence may be normed to obtain 
Banach spaces. We set $X_{k}$ to be the Banach space that results from norming $U_{S_{k}}$, and $Y_{k}$ the Banach space that results from norming $W_{S_{k}}$. Then we define spaces

$$
X:=\bigoplus_{n \in \mathbb{N}}^{p} X_{n}, \quad Y:=\bigoplus_{n \in \mathbb{N}}^{q} Y_{n}
$$

(where the choice of $1 \leq p, q \leq \infty$ doesn't matter; we may as well take $p=q=1$ ) and linear functions $T_{k}: X \rightarrow Y$ by

$$
T_{k}\left(\left(x_{m}\right)_{m \in \mathbb{N}}\right):=4^{k}\left(\delta_{k, m} \pi_{W_{S_{m}}}\left(x_{m}\right)\right)_{m \in \mathbb{N}},
$$

where

$$
\pi_{W_{S_{m}}}: U_{S_{m}} \rightarrow W_{S_{m}}
$$

are the canonical projections. Once more, considering the element $x=\left(x_{m}\right)_{m \in \mathbb{N}} \in X$ defined by the sequence

$$
x_{m}= \begin{cases}0 & k \neq m \\ \mathbf{1}_{\{\sigma\}} & k=m\end{cases}
$$

yields that the family of linear functions $\left(T_{k}\right)_{k \in \mathbb{N}}$ is unbounded, an we take the uniform boundedness principle to get a point $\left(x_{m}\right)_{m \in \mathbb{N}}$ such that

$$
\pi_{W_{S_{m}}}\left(x_{m}\right) \neq 0 \text { infinitely often. }
$$

By Lemma 3.3.11 this gives an infinite $I \subseteq \mathbb{N}$ and a family $\left(M_{n}\right)_{n \in I}$ of nonempty sets such that for all $n$ we have $M_{n} \subsetneq S_{n}$. Repeating this process $n$ times yields the theorem.

\subsection{The uniform boundedness principle for real-valued functions}

There is a weak form of the uniform boundedness principle that is equivalent (in the Zermelo-Fraenkel system) to the axiom of countable multiple choice:

3.4.1 Theorem (Weak form of the uniform boundedness principle) Let $X$ be a Banach space, and let $\left(T_{n}\right)_{n \in \mathbb{N}}$ be a sequence of linear and continuous functions from $X$ to $\mathbb{R}$ such that for all $x \in X$, the set

$$
\left\{T_{k}(x) \mid k \in \mathbb{N}\right\}
$$

is a bounded subset of $\mathbb{R}$. Then

$$
\sup _{k \in \mathbb{N}}\left\|T_{k}\right\|_{o p}<\infty .
$$

Journal of Logic \& Analysis 9:1 (2017) 
3.4.2 Theorem Assuming the Zermelo-Fraenkel system, but not any choice, Theorem 3.4.1 is equivalent to the axiom of countable multiple choice.

Proof For sufficiency, we note that the proof of Theorem 3.3.5 goes through without modification. For necessity, note that in our situation, we may modify the proof of Theorem 3.2.1 as follows so that only countable multiple choice is needed:

(1) The first use of the axiom of countable choice is avoided by taking the first element of the sequence $\left(T_{k}\right)_{k \in \mathbb{N}}$ which obeys the desired bound.

(2) The axiom of countable multiple choice is applied to get a finite subset $M_{n} \subset B_{n}$ ( $B_{n}$ as in the proof of Theorem 3.2.1) for each $n$, and if we set

$$
x_{n}:=\frac{1}{\# M_{n}} \sum_{x \in M_{n}} x,
$$

then $\left\|x_{n}\right\| \leq 1$ and $T_{n}\left(x_{n}\right)>\frac{2}{3}\left\|T_{n}\right\|_{o p}$.

\subsection{Outlook}

We have derived several choice-like axioms from the uniform boundedness principle. We believe that in conjunction, they are not strong enough to prove the axiom of countable choice, in particular in view of the fact that even if for each $n \in \mathbb{N}$ and each family $\left(S_{\alpha}\right)_{\alpha \in A}$ of sets of cardinality $n$

$$
\prod_{\alpha \in A} S_{\alpha} \neq 0
$$

the axiom of finite choice AC(fin) (see for instance Herrlich [15, Definition 2.6, page 14]) does not follow (see for instance Jech [15, Theorem 7.11, page 107]). We have been unable to deduce the axiom of countable choice from the uniform boundedness principle, but there are several lines of attack that seem promising:

(1) The use of a modified form of ultraproducts.

(2) The use of different characterisations of the uniform boundedness principle (for instance, as mentioned it is equivalent in $\mathrm{ZF}$ to the statement that all Banach spaces are barrelled).

(3) Using the fact that $Y$ merely needs to be a normed space.

Now the gap between what we proved and the axiom of countable choice is less huge than one would perhaps suspect; many choice axioms that we deduced from the uniform boundedness principle are of the 'partial type', but since partial countable choice 
is equivalent to countable choice, it would suffice to prove that from a sequence of nonempty sets $\left(S_{n}\right)_{n \in \mathbb{N}}$ that has arbitrary asymptotic behaviour, one can pick a sequence of subsets $\left(M_{n}\right)_{n \in \mathbb{N}}$ such that for infinitely many $n$ we have $\# M_{n}<C$ for a $C>0$ and $M_{n} \neq \emptyset$.

\section{Acknowledgements}

I would like to thank two users of a mathematical Q\&A website who helped me by explaining to me some facts about cardinalities. Furthermore, I would like to thank one of the referees for providing me with the wonderful and simple algebraic description of the spaces of Definition 3.3.10, which I had before described inefficiently (and not wholly correctly) by analytical means, and both referees for all the valuable suggestions they made. I would also like to thank the Editor for several explanations, and the Journal of Logic and Analysis for providing a venue without charge for papers of this kind.

\section{References}

[1] S Abbott, Understanding Analysis, volume 207 of Undergraduate Texts in Mathematics, 2 edition, Springer New York (2001); doi: 10.1007/978-1-4939-2712-8

[2] H L Bentley, H Herrlich, Countable choice and pseudometric spaces, Topology and its Applications 85 (1985) 153-164; doi: 10.1016/S0166-8641(97)00138-7

[3] N Bourbaki, Elements of mathematics. General topology. Part 2, Hermann, Paris; Addison-Wesley Publishing Co., Reading, Mass.-London-Don Mills, Ont. (1966)

[4] N Bourbaki, General Topology 1, volume 3 of Elements of Mathematics, 3 edition, Springer-Verlag Berlin Heidelberg (1995); doi: 10.1007/978-3-642-61701-0

[5] N Bourbaki, Topological Vector Spaces, volume 5 of Elements of Mathematics, Springer Berlin Heidelberg (2003); doi: 10.1007/978-3-642-61715-7

[6] H Brezis, Functional Analysis, Sobolev Spaces and Partial Differential Equations, 1 edition, Universitext, Springer New York (2011); doi: 10.1007/978-0-387-70914-7

[7] N Brunner, Garnir's dream spaces with Hamel bases, Archiv für mathematische Logik und Grundlagenforschung 26 (1987) 123-126; doi: 10.1007/BF02017496

[8] G Gutierres, On countable choice and sequential spaces, Mathematical Logic Quarterly 54 (2008) 145-152; doi: 10.1002/malq.200710018

[9] P R Halmos, Naive Set Theory, Undergraduate Texts in Mathematics, Springer New York (1974); doi: 10.1007/978-1-4757-1645-0 
[10] A Y Helemskii, Lectures and Exercises on Functional Analysis, volume 233 of Translations of Mathematical Monographs, American Mathematical Society (2006)

[11] H Herrlich, The Ascoli Theorem is equivalent to the Boolean Prime Ideal Theorem, Rostocker Mathematisches Kolloquium 51 (1997) 137-140; url: http://ftp.math.unirostock.de/pub/romako/heft51/herrlich.ps.Z

[12] H Herrlich, Axiom of Choice, Lecture Notes in Mathematics, Springer Berlin Heidelberg (2006); doi: 10.1007/11601562

[13] H Herrlich, GE Strecker, When is $\mathbb{N}$ Lindelöf?, Commentationes Mathematicae Universitatis Carolinae $38 \quad$ (1997) 553-556; url: http://dml.cz/bitstream/handle/10338.dmlcz/118952/CommentatMathUnivCarolRetro_381997-3_11.pdf

[14] M Hušek, Extension of Mappings and Pseudometrics, Extracta Mathematicae 25 (2010) 277-308; url: http://www.eweb.unex.es/eweb/extracta/Vol-25-3/25J3Husek.pdf

[15] T J Jech, The Axiom of Choice, volume 75 of Studies in Logic and the Foundations of Mathematics, North Holland (1973); doi: 10.1016/S0049-237X(08)71759-2

[16] K Keremedis, Disasters in topology without the axiom of choice, Arch. Math. Logic 40 (2001) 569-580; doi: 10.1007/s001530100094

[17] M Reimer, Multivariate Polynomial Approximation, volume 144 of International Series of Numerical Mathematics, Springer Basel (2003); doi: 10.1007/978-3-0348-8095-4

[18] Y T Rhineghost, The Naturals are Lindelöf iff Ascoli Holds, from: "Categorical Perspectives", (J Koslowski, A Melton, editors), Trends in Mathematics, Birkhäuser Boston (2001) 191-194; doi: 10.1007/978-1-4612-1370-3_11

[19] A P Robertson, W Robertson, On The Closed Graph Theorem, Glasgow Mathematical Journal 3 (1956) 9-12; doi: 10.1017/S2040618500033372

[20] W Rudin, Principles of Mathematical Analysis, 3 edition, International Series in Pure \& Applied Mathematics, McGraw-Hill (1976)

[21] E Schechter, Handbook of Analysis and Its Foundations, Elsevier (1997)

[22] AD Sokal, A really simple elementary proof of the uniform boundedness theorem, The American Mathematical Monthly 118 (2011) 450-452; doi: 10.4169/amer.math.monthly.118.05.450

[23] T Tao, An Epsilon of Room, I: Real Analysis, Graduate Studies in Mathematics 117, American Mathematical Society (2010); url: https://terrytao.files.wordpress.com/2012/12/gsm-117-tao3-epsilon1.pdf

[24] T Tao, An introduction to measure theory, Graduate Studies in Mathematics 126, American Mathematical Society (2011); url: https://terrytao.files.wordpress.com/2012/12/gsm126-tao5-measure-book.pdf

[25] K Yosida, Functional Analysis, 6 edition, Classics in Mathematics, Springer Berlin Heidelberg (1995); doi: 10.1007/978-3-642-61859-8 
25 Giggenhauser Road, 85354 Freising, DE

afellhau@netcologne.de

Received: 15 September $2015 \quad$ Revised: 21 November 2016 